\title{
Effects of alcohol, cigarettes, methamphetamine and marijuana exposure during pregnancy on maternal serum alpha-fetoprotein levels at 20-24 weeks'gestation
}

\begin{abstract}
Objective: To examine the effects of cigarette, marihuana and methamphetamine smoking and consumption of alcohol during pregnancy on maternal serum alpha-fetoprotein (MSAFP) levels at 20-24 weeks.

Study design: In the Safe Passage Study (SPS) more than 12,000 pregnant women were prospectively followed up during pregnancy and until the infant was one year old to examine the effects of exposure to alcohol during pregnancy on stillbirth and sudden infant death syndrome. The present study is a cross-sectional secondary analysis of MSAFP analyses done at 20-24 weeks gestation in 1,679 SPS participants, recruited at the Bishop Lavis Community Health Centre, Cape Town, South Arica.

Results: Low or moderate alcohol consumption with or without smoking, nor methamphetamine or marihuana use affected mean MSAFP levels. High MSAFP levels were associated with high alcohol consumption, young age, low body mass index (BMI) $\left(<18 \mathrm{~kg} / \mathrm{m}^{2}\right)$ or small mid upper arm circumference (MUAC) $(<230 \mathrm{~mm})$. High MSAFP levels were associated with stillbirth, preterm birth, abruption and a birth weight z-score of less than -1

Conclusion: The study confirms the association between high MSAFP levels and adverse pregnancy outcomes but, although exposure to smoking or drinking is associated with adverse pregnancy outcomes including stillbirth, MSAFP levels were not affected by any of these exposures except for continued high consumption of alcohol. The observed association between higher MSAFP levels and maternal nutritional status (as demonstrated by the lower MUAC and BMI) could explain some of the correlations of poor socioeconomic conditions with higher stillbirth rates effect.
\end{abstract}

Keywords: alpha-fetoprotein, tobacco, alcohol, pregnancy, stillbirth, placental abruption, preterm birth, birth weight, $\mathrm{Z}$ scores
Research Article

Volume 8 Issue I - 2018

Odendaal HJ,' L Geerts, ' DG Nel, ${ }^{2}$ LT Brink,' E Hitchcock, ${ }^{3}$ CA Groenewald'

'Department of Obstetrics and Gynecology, Faculty of Medicine and Health Science, Stellenbosch University, South Africa

${ }^{2}$ Department of Statistics and Actuarial Science, Stellenbosch University, South Africa

${ }^{3}$ Path Care Laboratories, Cape Town, South Africa

Correspondence: HJ Odendaal, Department of Obstetrics and Gynaecology, Stellenbosch University, South Africa, Email hjo@sun.ac.za

Received: January 29, 2018 | Published: February 27, 2018

\section{Introduction}

Addressing the burden of stillbirths is a worldwide priority. ${ }^{1}$ As many stillbirths are not associated with known risk factors, including previous obstetric history in primigravidas, ${ }^{2}$ the value of routine blood tests such as maternal serum alpha-fetoprotein (MSAFP) to identify pregnant women at risk should be explored. Although the main indication for MSAFP analyses in the mid-trimester is the exclusion of fetal neural tube defects, ${ }^{3}$ unexplained raised values are associated with stillbirths, ${ }^{4}$ low birth weight and fetal growth restriction ${ }^{5-8}$ and preterm delivery. ${ }^{9,10}$ In addition, elevated MSAFP levels., ${ }^{9,11,12}$ have also been associated with placental abruption, one of the main causes of stillbirth in high $^{13}$ and low-income countries ${ }^{14}$ or in mixed populations. ${ }^{15}$ Alcohol use during pregnancy may be associated with placental abruption, ${ }^{16-18}$ stillbirth, ${ }^{15}$ and small-for-gestational-age newborns. ${ }^{19}$ Cigarette smoking during pregnancy has also been associated with placental abruption, ${ }^{20}$ stillbirth ${ }^{21}$ and fetal growth restriction. ${ }^{22}$ It is therefore essential to know how environmental conditions such as smoking, drinking and the use of marijuana and methamphetamine affect MSAFP levels, both in pregnancies with normal and adverse outcomes, to improve interpretation of unexplained raised MSAFPvalues. The Safe Passage Study (SPS) of the Prenatal Alcohol in SIDS and Stillbirth (PASS) Network was undertaken to test the hypothesis that prenatal alcohol exposure is associated with increased risk for stillbirth and/or sudden infant death syndrome (SIDS) ${ }^{23}$ Detailed evaluations, including MSAFP, were performed during pregnancy to assess fetal growth and placental function. Information from the SPS would therefore provide valuable information on the interpretation and significance of MSAFP values.

\section{Materials and methods}

The information we obtained was under the auspices of SPS. Participants for the South African arm of the study were recruited at 
the antenatal clinic of the Bishop Lavis Community Health Centre, the primary health care centre for a low to middle class community. All study assessments at 20 weeks 0 days to 23 weeks 6 days $\left(20^{\circ}-23^{6}\right)$, $28^{\circ}-31^{6}$ and $34^{\circ}-37^{6}$ weeks' gestation were performed at Tygerberg Academic Hospital, Cape Town, South Africa. Recruitment for this prospective study began on 1 August 2007 and collection of blood for MSAFP was discontinued on 13 June 2011 (to limit the expenses of the study). A total of 1,679 singleton pregnancies, with MSAFP determined at $20^{\circ}-23^{6}$ weeks (the first SPS study visit) and known birth outcome, were included for this analysis (Figure 1). Gestational age (GA) was determined by ultrasound at the first antenatal visit or just before the second visit. ${ }^{23}$ After the recruitment interview, a trained midwife measured the weight, height and mid upper arm circumference (MUAC) of the participant and calculated the body mass index $\left(\mathrm{BMI}\right.$ in $\left.\mathrm{kg} / \mathrm{m}^{2}\right)$. A regularly calibrated high quality scale was used to determine weights after participants took off their heavy clothes, shoes and head dresses. A Seca stadiometer ${ }^{\circledR}$ (Baseline Scales and Balances (Pty) Ltd, 11 Wimbledon Road, Edgemead, 7447, Cape Town, South Africa), fixed against the wall, was used to measure the height. For the MUAC the midpoint between the acromion of the scapula and olecranon of the ulna was first determined and the circumference measured. This procedure was repeated from the beginning and the mean MUAC was used when the two measurements differed by less than $10 \%$. If the two measurements differed by more than $10 \%$, a third measurement was taken and the mean of the closest two measurements was used (the effect of the outlier was therefore excluded). Four millilitres maternal blood was taken for MSAFP level at the $20^{\circ}$ to $23^{6}$ weeks fetal assessment visit. Although in the clinical setting blood for MSAFP is usually taken at 15-22 weeks' gestation ${ }^{24}$ our sampling period ended 2 weeks later to suit the SPS study protocol. Blood was collected in a sterile plain specimen tube and immediately placed in a refrigerator until the blood of the last participant for the day was collected (always before midday) and then transported by a designated driver to Path Care laboratory where the MSAFP level was determined with standard methods (Chemiluminescence, Beckman Coulter Access Immunoassay ${ }^{\mathbb{}}$ ). Socioeconomic data, information on complications during pregnancy, GA at delivery, and fetal anthropometry were obtained from the SPS database and maternal chart abstraction after delivery. The placenta was examined by the midwife in charge of the delivery and information was entered in the medical record, where there was an allocated field to record presence and size of any placental abruption (size expressed as a percentage of the placental surface). In case of fetal demise the mother was approached for consent for autopsy. ${ }^{25}$

Causes of stillbirths were classified according to a classification designed for the SPS study. ${ }^{26}$ The MSAFP values were kept blind from the clinicians and the absolute MSAFP values were used for comparisons. In clinical practice, when screening for T21 or for neural tube defects, one uses multiples of the median (MoM) MSAFP values, corrected for factors like weight, race and diabetes as these affect AFP values but not the conditions screened for. In this study we did not use MoMs as several of these confounders may also affect the outcomes of interest and the GA at MSAFP collection was equally distributed amongst the different outcome categories. Information on alcohol and cigarette use was obtained at the recruitment interview, at up to three prenatal visits after recruitment, and at 1 month post-delivery. We used a modified timeline follow-back ${ }^{27}$ interview to determine alcohol exposure and self-reported frequency and quantity of tobacco cigarettes use for smoking exposure. Drinks per drinking day and cigarettes per day were calculated for each month of pregnancy for which exposure information was available. Group-based trajectory modelling was used to categorize pregnancies with similar drinking and smoking exposure patterns incorporating quantity, frequency and timing of exposure during pregnancy, adjusted for number of days' exposure. ${ }^{28}$ There were five distinct drinking trajectories characterized as none, moderate quit early, high quit later, low continuous and high continuous. Seven distinct smoking trajectories were identified and characterized as none, moderate quit early, high quit later, low continuous, moderate continuous, high continuous and very high continuous. The drinking and smoking trajectories were dichotomized separately: no drinking or smoking (none, quit early defined as no exposure during pregnancy or cessation around the end of the first trimester) and drinking or smoking (continuous, quit late defined as continuous exposure throughout pregnancy or cessation beyond the first trimester). To examine dual exposure, a four-level exposure variable was created:

\section{i. No drinking and no smoking, \\ ii. Smoking only,}

iii. Drinking only,

iv. Drinking and smoking.

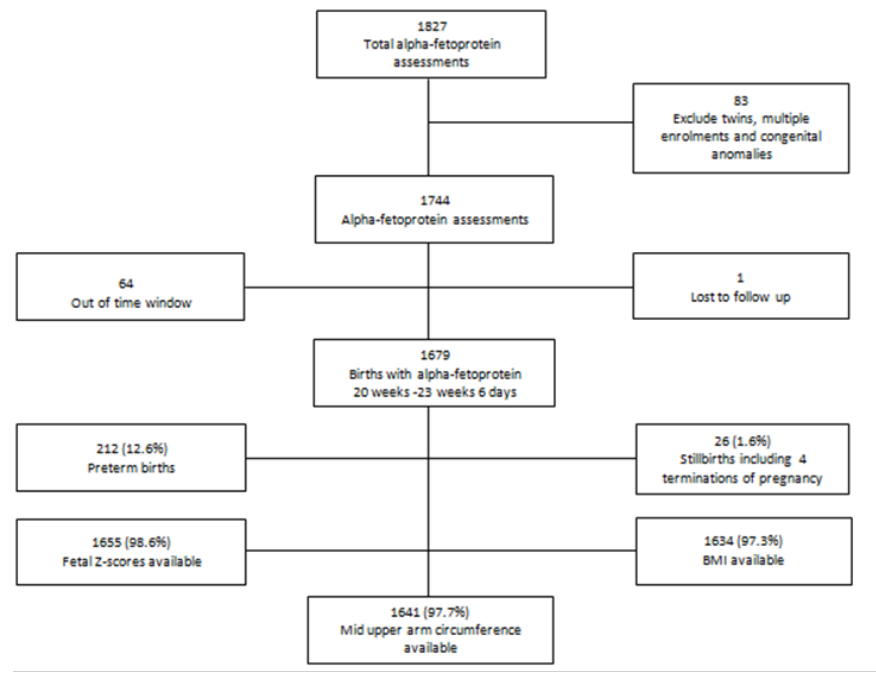

\section{Figure I Study profile}

The exposure to marijuana and methamphetamine was not quantified; only its use during pregnancy was recorded. Other information collected included stillbirth, preterm delivery (any delivery before 259 days, either spontaneous or iatrogenic), hypertensive disease (including pre-existing hypertension, pregnancyinduced hypertension, preeclampsia and eclampsia), maternal age, fetal sex, and Z-scores of birth weight for GA, calculated from the international standards of the INTERGROWTH- $21^{\text {st }}$ study (available for gestational ages from 168-299 days). ${ }^{29}$ Written informed consent was obtained before recruitment to the study. Ethical approval has been obtained from the Health Research Ethics Committee of Stellenbosch University (Ethics approval number: N06/10/210). 
Statistical analyses. All the information was entered on an Excel spread sheet. Statistical analyses were done with STATISTICA (Dell Inc. (2016) Dell Statistica ${ }^{\circledR}$ (data analysis software system), version 13. software.dell.com). At first, descriptive analyses were performed to identify outliers. Continuous variables were compared between groups with analysis of variance (ANOVA). Bonferroni or least significant difference (LSD) multiple comparisons were used to identify significant differences between the means. Spearman correlations were used to measure correlation between repetitions of several response variables. Intra-class correlations for agreement and consistency were also calculated.

\section{Results}

There were 1,679 participants in the study (Figure 1) and patient characteristics are presented in Table 1. The mean age at recruitment was 24.6 years and the majority of women were parous. The mean BMI was $25.4 \mathrm{~kg} / \mathrm{m}^{2} ; 43 \%$ of women were obese. Social circumstances were often poor. The median number of people per room living in the home was 1.3 (0.3 to 9.0$)$ and only $24 \%$ of participants lived in a council house while the majority $(67.4 \%)$ lived in informal settlements and the remaining $8.6 \%$ in flats, mobile homes and trailers or on farms. Half $(50 \%)$ of the participants were partnered but living apart, $24.1 \%$ were married, $19.1 \%$ were partnered and living together and the remaining $6.8 \%$ were single, divorced or widowed. The mean GA at enrolment was 120.0 days (17 weeks 1 day) and the GA at blood collection for MSAFP ranged from 140 to 167 days with a mean of 157.6 days. MSAFP-values ranged from 19.2 to $665.1 \mathrm{IU} / \mathrm{mL}$ with a mean of $86.7 \mathrm{IU} / \mathrm{mL}$ (Table 1). Table 2 shows the distribution of drinking and smoking trajectories: Only $47 \%$ of pregnant women did not smoke cigarettes during the pregnancy or stopped early and $63 \%$ did not drink or stopped early. Table 3 compares absolute MSAFP values based on historical risk factors for adverse outcome or exposure history. MSAFP values did not differ significantly between pregnancies with or without medical or obstetric risk factors but we noted significantly lower MSAFP values in women with gestational hypertension $(p<0.01)$ and significantly higher values in women younger than 20 years and in male fetuses in spite of very similar mean GA at MSAFP collection for these different categories (two days or less difference) (Table 6). No significant differences in mean MSAFP values were seen across the five drinking trajectory groups (Figure 2, $\mathrm{p}>0.05$ ) but when drinking trajectories were collapsed into two groups, there was a significantly higher mean MSAFP value amongst heavy continuous drinkers (trajectory 5) compared to other drinking patterns (trajectories 1-4) $(\mathrm{p}<0.001$, Table 3$)$ at an identical mean GA at MSAFP collection (158 days) (Table 6). No significant differences in MSAFP values were seen across the seven smoking trajectory groups (LS means current effect: $F(6,1736)=0.20587, p=0.98$, KruskalWallis $\mathrm{p}>0.05$ ) or with exposure to marihuana or methamphetamine.. There were significant differences in absolute MSAFP values (at very similar GA) according to maternal size (Table 4). MSAFP values decreased progressively with increasing $\operatorname{MUAC}(\mathrm{p}<0.0001$, Figure 3$)$ and were significantly lower in women with high BMI $\left(>25 \mathrm{~kg} / \mathrm{m}^{2}\right)$ compared to those with normal $\left(18-25 \mathrm{~kg} / \mathrm{m}^{2}\right)$ or low $\left(<18 \mathrm{~kg} / \mathrm{m}^{2}\right)$ BMI ( $<0.0001$, Figure 4) (no significant difference between those with normal and low BMI). In addition, no significant differences in MSAFP values were demonstrated when comparing any drinking during pregnancy to no drinking, any smoking during pregnancy to no smoking, smoking only, drinking only and dual exposure compared to no drinking (Table 4). Increasing MSAFP values were associated with progressively smaller fetal size: mean MSAFP values (at very similar GA of sampling) differed significantly across the $4 \mathrm{Z}$-score groups ( $\mathrm{p}<$ 0.001 , Table 4, Figure 5).

Pregnancy outcome data are displayed in Table 5 and mean GA at MSAFP sample collection was similar for women with and without complications. Twelve women developed placental abruption with a mean interval between MSAFP sample collection and delivery of 74.1 days (range 6 to 134 days). Their mean MSAFP values were significantly higher than for women without abruption $(p<0.05)$. Stillbirths occurred in 26 pregnancies with a mean interval between MSAFP sample collection and delivery of 61.3 days (range 6 to 133 days). The mean MSAFP values preceding stillbirth were significantly higher than those preceding live births $(p<0.0001)$. Preterm births occurred in 212 pregnancies at a mean interval since MSAFP sample collection of 80.1 days (range 6 to 117 days). Mean MSAFP values preceding preterm birth were significantly higher than for term births $(p<0.0001)$. There was a significant correlation between the MSAFP value and test-delivery time $(\mathrm{r}=-0.2769, \mathrm{p}<0.0001)$ overall, with longer intervals associated with lower MSAFP values (Table 6).

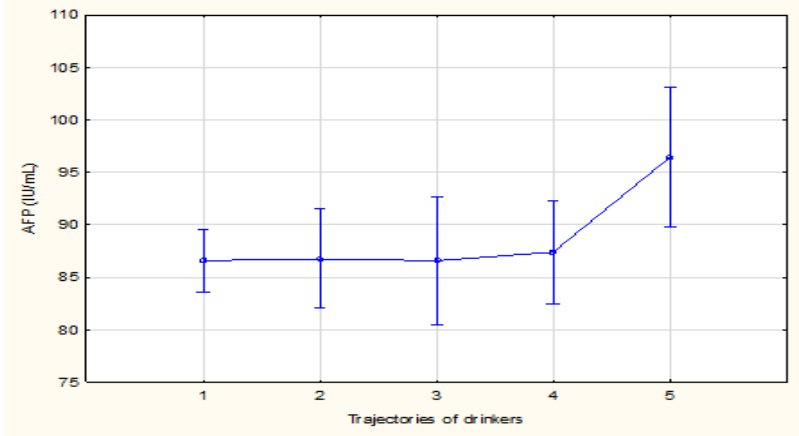

Figure 2 Effect of alcohol trajectory on mean maternal serum alphafetoprotein values

LS means current effect: $F$ (4, I738), I.8834; p, 0.I I; Kruskal-Wallis; p, 0.17 I, none; 2 , moderate quit early; 3 ,high quit later; 4 , low continuous; 5 , high continuous. Vertical bars denote $95 \%$ confidence intervals.

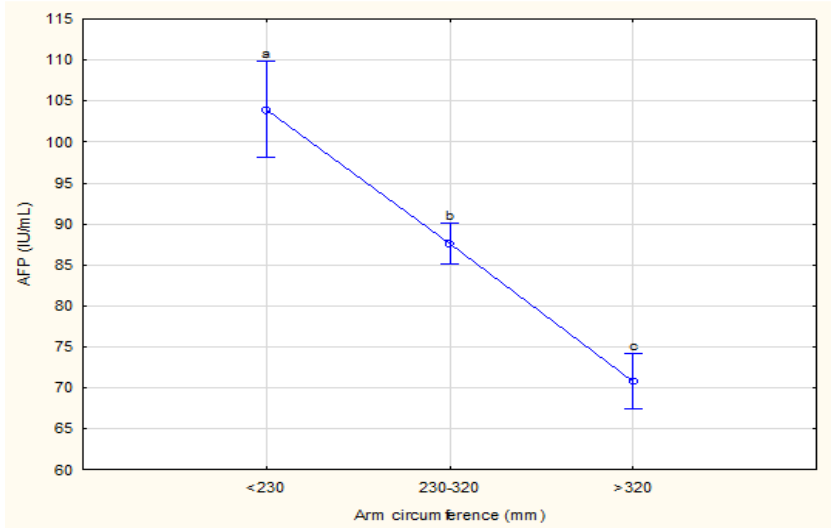

Figure 3 Effect of mid upper arm circumference on mean maternal serum alpha-fetoprotein levels

Weighted means current effect: F (2, I70I), 40.533; $p<0.000$ I. Vertical bars denote $95 \%$ confidence intervals. Different letters above the vertical bars indicate significant differences, in this figure between all variables 
Table I Descriptive Statistics

\begin{tabular}{|c|c|c|}
\hline Variable & $\mathrm{N}$ & Mean \pm SD or Median (Range) \\
\hline Maternal age (years) & 1679 & $24.6 \pm 5.9(16-43)$ \\
\hline Gravidity & 1679 & $2(1-8)$ \\
\hline Parity & 1679 & I (0-7) \\
\hline BMI $\left(\mathrm{kg} / \mathrm{m}^{2}\right)$ & 1634 & $25.4 \pm 5.8(14.8-55.9)$ \\
\hline GA at enrollment (days) & 1679 & $120.0 \pm 29.3(43-167)$ \\
\hline MUAC (mm) & $|64|$ & $278.5 \pm 47.2(184.5-477)$ \\
\hline Rooms in house & 1668 & $4(I-I I)$ \\
\hline People in house & 1678 & $5(I-22)$ \\
\hline People per room & 1668 & I.3 (0.3-9) \\
\hline GA at MSAFP collection (days) & 1679 & $157.6 \pm 6.9(140-167)$ \\
\hline MSAFP (IU/mL) & 1679 & $86.7 \pm 41.9(19.2-665.1)$ \\
\hline
\end{tabular}

N, Number; BMI, Body mass index; GA, Gestational age; MUAC, Mid upper arm circumference; MSAFP Maternal serum alpha-fetoprotein; SD, Standard deviation.

Table 2 Distribution of drinking and smoking trajectories

\begin{tabular}{cccccccc}
\hline Trajectory & I & 2 & 3 & 4 & 5 & 6 & 7 \\
\hline Smoking N (\%) & $65 I(39)$ & $129(8)$ & $36(2)$ & $264(16)$ & $415(25)$ & $156(9)$ & $28(2)$ \\
Drinking N (\%) & $755(45)$ & $306(18)$ & $181(11)$ & $281(17)$ & $156(9)$ & - & - \\
\hline
\end{tabular}

Table 3 Effects of medical and obstetric history, maternal age, fatal sex and use of alcohol, cigarettes, marihuana and methamphetamine on mean maternal serum alpha-fetoprotein levels \pm SD (in IU/mL)

\begin{tabular}{|c|c|c|c|}
\hline Variable & No & Yes & p-value \\
\hline \multicolumn{4}{|l|}{ Abnormal Medical history } \\
\hline N (\%) & $1020(61)$ & 658 (39) & \\
\hline Mean MSAFP \pm SD $(95 \% \mathrm{Cl})$ & $86.7 \pm 41.1(84.2-89.3)$ & $86.5 \pm 43.1(83.2-89.8)$ & $>0.05^{*}$ \\
\hline \multicolumn{4}{|l|}{ Previous miscarriage } \\
\hline $\mathrm{N}(\%)$ & $857(85)$ & $157(15)$ & \\
\hline Mean MSAFP \pm SD $(95 \% \mathrm{Cl})$ & $85.4 \pm 37.6(82.9-87.9)$ & $84.7 \pm 55.4(76.0-93.5)$ & $>0.05^{*}$ \\
\hline \multicolumn{4}{|l|}{ Previous stillbirth } \\
\hline $\mathrm{N}(\%)$ & $979(97)$ & $35(3)$ & \\
\hline Mean MSAFP \pm SD $(95 \% \mathrm{Cl})$ & $85.3 \pm 4 I . I(82.8-87.9)$ & $83.9 \pm 34.0(72.2-95.6)$ & $>0.05^{*}$ \\
\hline \multicolumn{4}{|l|}{ Gestational hypertension } \\
\hline $\mathrm{N}(\%)$ & $1522(96)$ & $66(4)$ & \\
\hline Mean MSAFP \pm SD $(95 \% \mathrm{Cl})$ & $87.0 \pm 42.0(84.9-89.1)$ & $72.5 \pm 34.3(64.1-81.0)$ & $<0.01 *$ \\
\hline \multicolumn{4}{|l|}{ Maternal age $<20$ years } \\
\hline $\mathrm{N}(\%)$ & 1298 (77.3) & $38 \mathrm{I}(22.7)$ & \\
\hline Mean MSAFP \pm SD $(95 \% \mathrm{Cl})$ & $85.5 \pm 37.9(83.4-87.6)$ & $90.6 \pm 53.0(85.3-96.0)$ & $<0.05 \#$ \\
\hline \multicolumn{4}{|l|}{ Male fetus } \\
\hline N (\%) & $840(50.6)$ & $821(49.4)$ & \\
\hline
\end{tabular}

Citation: Odendaal HJ, Geerts L, Nel DG, et al. Effects of alcohol, cigarettes, methamphetamine and marijuana exposure during pregnancy on maternal serum alpha-fetoprotein levels at 20-24 weeks'gestation.J Pediatr Neonatal Care. 2018;8(I): 003 I4. DOI: 10.15406/jpnc.2018.08.003 |4 
Table Continued.....

\begin{tabular}{|c|c|c|c|}
\hline Variable & No & Yes & p-value \\
\hline Mean MSAFP \pm SD $(95 \% \mathrm{Cl})$ & $84.6 \pm 44.7(81.6-87.7)$ & $88.5 \pm 37.6(85.9-91.1)$ & $<0.0 I^{*}$ \\
\hline \multicolumn{4}{|c|}{ Trajectory 5 Drinking during pregnancy } \\
\hline $\mathrm{N}(\%)$ & $1523(9 \mid)$ & $156(10)$ & \\
\hline Mean MSAFP \pm SD $(95 \% \mathrm{Cl})$ & $85.6 \pm 39.2(83.6-87.6)$ & $97.2 \pm 61.5(87.5-106.9)$ & $<0.001 \#$ \\
\hline \multicolumn{4}{|c|}{ Trajectory 5-7 Smoking during pregnancy } \\
\hline $\mathrm{N}(\%)$ & $1080(64)$ & $599(36)$ & \\
\hline Mean MSAFP \pm SD $(95 \% \mathrm{Cl})$ & $85.7 \pm 39.9(83.3-88.1)$ & $88.3 \pm 45.1(84.7-92.0)$ & $>0.05 \#$ \\
\hline \multicolumn{4}{|l|}{ Any marijuana during pregnancy } \\
\hline$N(\%)$ & $1510(91)$ & $142(9)$ & \\
\hline Mean MSAFP \pm SD $(95 \% \mathrm{Cl})$ & $86.1 \pm 39.9(84.1-88.1)$ & $91.3 \pm 58.3(81.6-100.9)$ & $>0.05^{*}$ \\
\hline \multicolumn{4}{|c|}{ Any methamphetamine during pregnancy } \\
\hline $\mathrm{N}(\%)$ & $1569(95)$ & $82(5)$ & \\
\hline Mean MSAFP \pm SD $(95 \% \mathrm{Cl})$ & $86.3 \pm 42.0(84.2-88.3)$ & $91.5 \pm 38.5(83.1-100.0)$ & $>0.05^{*}$ \\
\hline
\end{tabular}

N, Number; MSAFP, Maternal serum alpha-fetoprotein; SD, Standard deviation; Cl, Confidence interval; *p-value with Mann-Whitney U; \# p-value with LS mean

Table 4 Effects of mid upper arm circumference, body mass index, smoking and drinking combinations and birth weight z-scores on mean maternal serum alpha-fetoprotein levels \pm SD (in IU/mL)

\begin{tabular}{|c|c|c|c|}
\hline Variable & $\mathrm{N}(\%)$ & Mean MSAFP \pm SD $(95 \% \mathrm{Cl})(\mathrm{IU} / \mathrm{ml})$ & $P$ \\
\hline \multicolumn{3}{|c|}{ Mid Upper Arm Circumference } & $<0.000 I^{*}$ \\
\hline$<230 \mathrm{~mm}$ & $208(12.7)$ & $104.0 \pm 42.8(98.1-109.8)$ & \\
\hline $230-320 \mathrm{~mm}$ & II $23(68.4)$ & $87.6 \pm 42.9(85.1-90.1)$ & \\
\hline$>320 \mathrm{~mm}$ & $310(18.9)$ & $70.8 \pm 30.2(67.4-74.2)$ & \\
\hline \multicolumn{3}{|l|}{ Body mass index } & $<0.000$ I* \\
\hline$<18 \mathrm{~kg} / \mathrm{m}^{2}$ & $64(3.9)$ & $105.4 \pm 36.7(96.2-114.5)$ & \\
\hline $18-25 \mathrm{~kg} / \mathrm{m}^{2}$ & $875(53.7)$ & $93.8 \pm 43.1(90.9-96.7)$ & \\
\hline$>25 \mathrm{~kg} / \mathrm{m}^{2}$ & $695(42.5)$ & $76.1 \pm 38.9(73.2-79.0)$ & \\
\hline \multicolumn{3}{|c|}{ Smoking and drinking during pregnancy } & $>0.05 \#$ \\
\hline No & $622(37)$ & $86.1 \pm 43.5(82.7-89.5)$ & \\
\hline Smoking only & $439(26)$ & $84.7 \pm 33.8(81.5-87.8)$ & \\
\hline Drinking only & $158(9)$ & $85.8 \pm 35.5(80.2-91.4)$ & \\
\hline Both & $460(27)$ & $89.7 \pm 48.0(85.3-94.1)$ & \\
\hline \multicolumn{3}{|c|}{ Z-scores of birth weight } & $<0.000 I^{*}$ \\
\hline$<-1$ & $437(26.4)$ & $94.9 \pm 55.3(89.8-100.1)$ & \\
\hline-1 & $658(39.8)$ & $86.2 \pm 35.5(83.4-88.9)$ & \\
\hline $0-1$ & $4 \mid 4(25.0)$ & $83.8 \pm 34.2(80.5-87.1)$ & \\
\hline$>1$ & I 46 (8.8) & $71.5 \pm 295(66.7-76.3)$ & \\
\hline
\end{tabular}

N, Number; MSAFP, Maternal serum alpha-fetoprotein; SD, Standard deviation; Cl, Confidence interval; * p-value with weighted means test; \# p-value with 
Kruskal Wallis test

Table 5 Pregnancy complications related to mean maternal serum alpha-fetoprotein levels (in IU/mL)

\begin{tabular}{|c|c|c|c|}
\hline Variable & Yes & No & $\mathrm{P}$ \\
\hline Clinical abruption & & & $<0.05^{*}$ \\
\hline $\mathrm{N}(\%)$ & $12(0.7)$ & $1652(99.3)$ & \\
\hline Mean MSAFP \pm SD $(95 \% \mathrm{Cl})$ & III.I $\pm 68.3(67.7-154.4)$ & $86.4 \pm 4 I . I(84.4-88.4)$ & \\
\hline Stillbirth & & & $<0.000$ I* \\
\hline N (\%) & $26(1.6)$ & 1635 (98.4) & \\
\hline Mean MSAFP \pm SD $(95 \% \mathrm{Cl})$ & $149.0 \pm 158.6(84.9-213.0)$ & $85.6 \pm 35.9(83.8-87.3)$ & \\
\hline Preterm birth & & & $<0.000 I^{*}$ \\
\hline N (\%) & $212(12.6)$ & | 467 (87.4) & \\
\hline Mean MSAFP \pm SD $(95 \% \mathrm{Cl})$ & $101.7 \pm 67.0(92.6-110.7)$ & $84.5 \pm 36.4(82.6-86.4)$ & \\
\hline
\end{tabular}

N, Number; MSAFP, Maternal serum alpha-fetoprotein; SD, Standard deviation, ${ }^{*} \mathrm{p}$-values with weighted means test.

Table 6 Gestational ages when blood for AFP was taken in the different variables

\begin{tabular}{|c|c|c|}
\hline & $\mathrm{N}(\%)$ & Mean GA at MSAFP (days) \\
\hline \multicolumn{3}{|c|}{ Hypertensive disease during pregnancy } \\
\hline No & $1522(96)$ & 158 \\
\hline Yes & $66(4)$ & 157 \\
\hline \multicolumn{3}{|c|}{ Drinking during pregnancy } \\
\hline Traj I - 4 & $1523(91)$ & 158 \\
\hline Traj 5 & $156(10)$ & 158 \\
\hline \multicolumn{3}{|c|}{ MUAC (mm) } \\
\hline$<230$ & $208(12.7)$ & 159 \\
\hline $230-320$ & II $23(68.4)$ & 158 \\
\hline$>320$ & $310(18.9)$ & 157 \\
\hline \multicolumn{3}{|c|}{$\mathrm{BMI}\left(\mathrm{kg} / \mathrm{m}^{2}\right)$} \\
\hline$<18$ & $64(3.9)$ & 157 \\
\hline $18-25$ & $875(53.7)$ & 158 \\
\hline$>25$ & $695(42.5)$ & 157 \\
\hline \multicolumn{3}{|c|}{ Maternal age (years) } \\
\hline$<20$ & $38 \mathrm{I}(22.7)$ & 158 \\
\hline$\geq 20$ & $1298(77.3)$ & 157 \\
\hline \multicolumn{3}{|c|}{ Z-scores of birth weight } \\
\hline$<-1$ & $437(26.4)$ & 158 \\
\hline-1 & $658(39.8)$ & 158 \\
\hline $0-1$ & $4 \mid 4(25.0)$ & 158 \\
\hline$>1$ & $146(8.8)$ & 157 \\
\hline \multicolumn{3}{|c|}{ Clinical abruption } \\
\hline No & 1652 (99.3) & 158 \\
\hline Yes & $12(0.7)$ & 158 \\
\hline
\end{tabular}




\begin{tabular}{|c|c|c|}
\hline & N (\%) & Mean GA at MSAFP (days) \\
\hline \multicolumn{3}{|c|}{ Pregnancy outcome } \\
\hline Live birth & $1635(98.4)$ & 158 \\
\hline Stillbirth & $26(1.6)$ & 157 \\
\hline \multicolumn{3}{|c|}{ Preterm birth } \\
\hline No & | 467 (87.4) & 158 \\
\hline Yes & $212(12.6)$ & 157 \\
\hline \multicolumn{3}{|l|}{ Fetal sex } \\
\hline Female & $840(50.6)$ & 158 \\
\hline Male & $821(49.4)$ & 158 \\
\hline
\end{tabular}

N, Number; GA, gestational age; MUAC, mid Upper Arm Circumference (mm); BMI, Body Mass index $\left(\mathrm{kg} / \mathrm{m}^{2}\right)$.

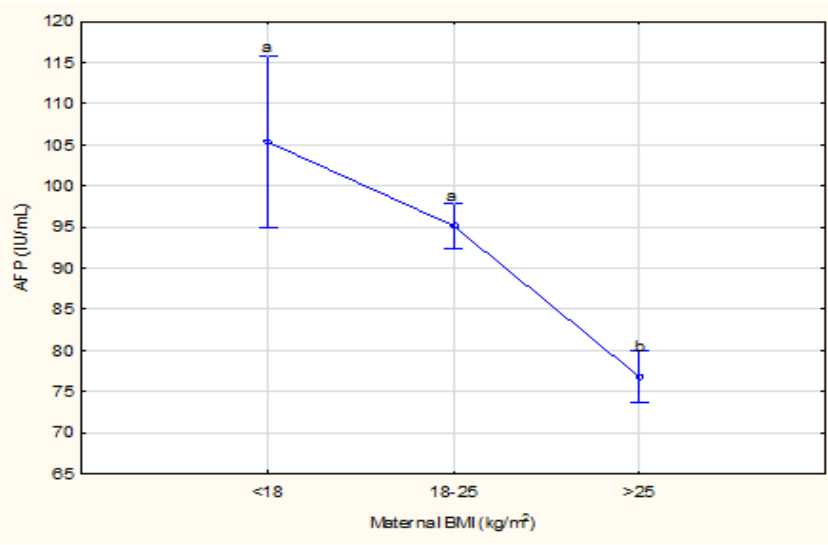

Figure 4 Effect of BMI at recruitment on mean maternal serum alphafetoprotein levels

Weighted means current effect: $F(2,1631), 42.714 ; p<0.0001$. Vertical bars denote $95 \%$ confidence intervals. Different letters above the vertical bars indicate significant differences, e.g. in this figure between the first 2 variables (a) and last one (b) Difference between the first 2 variables is not significant as both have the same letter (a) above the vertical bars.

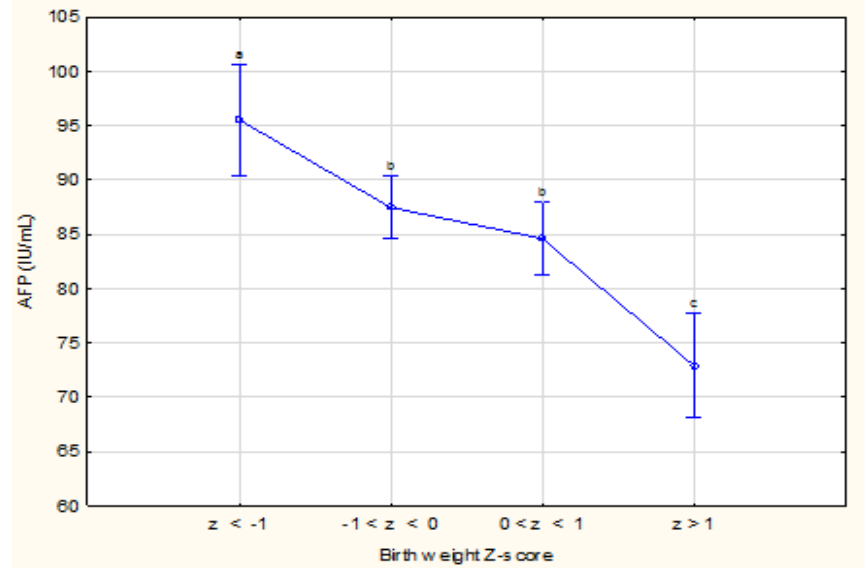

Figure 5 Association between Z-scores of birth weight and mean materna serum alpha-fetoprotein levels

Weighted means current effect: $F(3,165$ I), 13.363; $p<0.000$ I. Vertical bars denote $95 \%$ confidence intervals. Different letters above the vertical bars indicate significant differences, e.g. in this figure between the first variables (a), the second two (b) and last one (c). Difference between the middle 2 variables is not significant as both have the same letter (b) above the vertical bars.

\section{Discussion}

Very little is known about MSAFP values in drinkers but this study found raised values in heavy continuous drinkers. A small study in 1987 suggested the use of MSAFP, human placental lactogen and pregnancy-specific beta-1-glycoprotein to identify fetal alcohol syndrome in drinking women ${ }^{30}$ as the authors postulated that alcohol exposure could inhibit AFP production by the fetal liver. This suggestion was not pursued further but, based on the results of this current large cohort with detailed and prospective collection of alcohol exposure data, it seems reasonable to believe that heavy maternal alcohol exposure during pregnancy increases MSAFP levels when determined at 20-24 weeks gestation. We could not demonstrate any significant effect of smoking trajectory on the MSAFP levels. This is in contrast with Spencer ${ }^{31}$ who studied 30,727 self-reported smokers and non-smokers where the pregnancy resulted in the birth of a normal fetus. Median MSAFP values were 3\% higher in smokers but the degree of smoking was not quantified. Several other studies have also described the association between MSAFP levels and cigarette smoking. ${ }^{32,33}$ Our findings differ from those reported in the literature but as we collected the information on smoking prospectively on several occasions during pregnancy and compared distinctly different exposure groups, we have confidence in the quality of exposure information used for this analysis in contrast to studies where information on smoking was collected retrospectively. On the other hand, our study may have been underpowered to detect a 3\% difference in MSAFP levels especially in those continuing to smoke moderately or heavily. Alternatively, environmental smoking exposure may well have attenuated the effect of maternal smoking on MSAFP values in this study. This aspect was not specifically investigated in this study, but as there were up to 22 inhabitants in a house and up to 9 sharing a room, the effect of environmental exposure to cigarette smoke could have been high in this cohort and this may have masked the presumed protective effect of not smoking during pregnancy. In an observational study Heinonen et al. ${ }^{6}$ compared 123 pregnant smokers with unexplained elevated AFP levels (i.e. those not due to a fetal anomaly, incorrect gestational age, miscarriage or twins) in the second trimester with 827 smokers with normal MSAFP levels, 471 non-smokers with raised MSAFP values and 12,596 nonsmokers with normal MSAFP values. Raised values were associated with increased risk of small-for-gestational-age newborns, low birth weight and preterm birth. Although the authors did not specifically examine the effect of smoking on the MSAFP values themselves, they found that the effects of smoking and unexplained elevated MSAFP were additive in identifying the risk for poor outcome when both risk 
factors were present at the same time. The pathways through which smoking may affect the fetal liver was explored by Filis et al. ${ }^{34}$ on 24 post-termination liver specimens of morphologically normal foetuses. They found that maternal smoking affected fetal liver expression of proteins with roles in posttranslational protein processing and secretion, stress response, detoxification and homeostasis. Some of the affected pathways differed between male and female foetuses. We found significantly higher MSAFP values in pregnancies with male foetuses, in line with previous studies where MSAFP levels were $3 \%{ }^{35}$ and $6 \%{ }^{36}$ lower in euploid female compared to male fetuses.

No previous publications specifically reported the association between MSAFP levels and maternal BMI or MUAC but it is widely practiced to adjust MSAFP levels for maternal weight (to obtain MSAFP-MoM) when calculating the risk for a neural tube defect or Down syndrome in the fetus, to compensate for the assumed dilution effect of a larger blood volume with greater BMI. Our study demonstrated that, compared to a BMI $>25 \mathrm{~kg} / \mathrm{m}^{2}$, a low or normal BMI in early pregnancy was associated with higher MSAFP levels and especially participants with a BMI of $<18 \mathrm{~kg} / \mathrm{m}^{2}$ had significantly higher values. MUAC correlates closely with BMI and it has been reported that MUAC $<24 \mathrm{~cm}$ for a female may be a simpler alternative to a BMI cut-off $<18.5 \mathrm{~kg} / \mathrm{m}^{2}$ to detect adult undernutrition. ${ }^{37}$ Since we found such a good correlation between both a small MUAC and low BMI with higher MSAFP levels, one needs to consider the possibility of a nutritional factor affecting MSAFP levels, which may partly explain the higher rate of e.g. pre-labour stillbirths in low income countries. Although unexplained raised MSAFP levels have been reported in mothers who delivered low birth weight newborns, ${ }^{38,39}$ and small-for-gestational-age infants, ${ }^{7,6}$ this seems to be the first study comparing MSAFP levels directly to Z-scores of the birth weight We found that $Z$-scores $<-1$ or $>+1$ were associated with significantly higher and lower MSAFP levels respectively (difference between them of $23.7 \%$ ) when compared with Z-scores between -1 and +1 . The finding of lower MSAFP values in those with a birthweight $Z$-score of greater than +1 and the higher value with birthweight $Z$-scores less than -1 is in keeping with the known association between high MSAFP values and small for gestational age infants. High values should therefore be a cause for concern and an indication for careful follow-up during pregnancy.

We demonstrated a significant increase in mean MSAFP values in pregnancies where stillbirths occurred later in pregnancy. Unexplained raised MSAFP has previously been associated with higher risk of fetal death. ${ }^{8,40}$ Smith et al. ${ }^{4}$ studied more than 84,000 prim gravid women delivering an infant at or after 24 weeks of gestation. The odds ratio for stillbirth at 24-28 weeks with a MSAFP value greater than the $99^{\text {th }}$ percentile was 11.97 (95\% CI: $5.34-26.83$ ); for stillbirths at or after 37 weeks, the odds ratio was much lower. Predictability was generally poor except for demises at extreme preterm gestations. Raised MSAFP levels seem to be associated with an increased risk of placental abruption. ${ }^{11,39}$ Tikkanen et al. ${ }^{12}$ compared 57 women with and 108 without abruption regarding second trimester MSAFP values and found significantly higher levels in the abruption group. The very highest values were found amongst abruptions but there was a large overlap between cases and controls and the timing and outcome of the abruption was not mentioned. Although there were only 12 cases of placental abruption (11with acute and 1 with chronic abruption) in the current study, their MSAFP values were significantly higher when compared to pregnancies without abruption. The raised MSAFP at 2024 weeks in women who later developed abruption is probably an indication of poor placentation. In a previous study of the SPS cohort we found a good correlation between GA calculation from foot length at autopsy and early ultrasound in 12 fetuses where abruption was the cause of death ${ }^{41}$ indicating that it was unlikely that these fetuses were severely growth restricted or that the abruptions occurred long before delivery.

In a large population-based study of more than 100000 singleton pregnancies, women with a raised $\operatorname{MSAFP}(\geq 2.0 \mathrm{MoM})$ were at greater risk of preterm birth. ${ }^{10}$ Other studies also found this association ${ }^{6,7,38}$ and we have confirmed this and further demonstrated a strong inverse correlation between the test-delivery-interval and MSAFP level, with lower values associated with longer intervals. The significant inverse correlation between the test-delivery-interval and MSAFP value probably reflects the combined increased likelihood of preterm delivery, fetal demise or placental abruption, as the main reasons for an elevated MSAFP value. This confirms the need for careful followup of pregnancies with unexplained high values for complications resulting in either spontaneous or iatrogenic (medically-indicated) preterm birth. The study could be criticized in that many specimens (44\%) were taken later than the 22 weeks used as the upper GA limit in most prenatal screening programmes. It has been shown however, that serum screening for e.g. Down syndrome is also possible between 19 and 24 weeks $^{40}$ or from 18 to 35 weeks. ${ }^{42}$ The effect of environmental smoking was not specifically investigated in this cohort and may well have attenuated the effect of direct maternal smoking on MSAFP values seen in other studies, as living conditions in this cohort were most likely very different from previous reports. This may limit the generalisability of our finding that MSAFP levels were not affected by smoking trajectory. ${ }^{43}$

\section{Conclusion}

In this unselected pregnant population, with a high prevalence of smoking and/or drinking during pregnancy and with prospective collection of information on exposure, we found that raised MSAFP levels correlated significantly with heavy continuous drinking during pregnancy and with preterm labour, stillbirth, placental abruption and fetal growth deficit. The association of low birth weight Z-scores, small MUAC and low maternal BMI with higher MSAFP values raises the question of the role of nutrition in unexplained raised MSAFP levels and its known associations with adverse pregnancy outcomes. Pregnant women with unexplained raised MSAFP values should be carefully followed up during pregnancy.

\section{Acknowledgement}

The study was funded by the National Institute on Alcohol Abuse and Alcoholism, Eunice Kennedy Shriver National Institute of Child Health and Human Development, and National Institute on Deafness and Other Communication Disorders: U01 HD055154, U01 HD045935, U01 HD055155, U01 HD045991, and U01 AA016501.

\section{Conflict of interest}

The authors have no conflict to declare. 


\section{Referencess}

1. Frøen JF, Cacciatore J, McClure EM, et al. Stillbirths: Why they matter. Lancet 2011;377(9774):1353-1366.

2. Stillbirth Collaborative Research Network Writing Group. Causes of death among stillbirths. JAMA. 201;306(22):2459-2468.

3. Brock D, Scrimgeour J, Steven J, et al. Maternal plasma alpha-fetoprotein screening for fetal neural tube defects. BJOG: Int J Obstet Gynaecol. 1978;85(8):575-581

4. Smith GCS, Shah I, White IR, et al. Maternal and biochemical predictors of antepartum stillbirth among nulliparous women in relation to gestational age of fetal death. BJOG Int J Obstet Gynaecol. 2007;114(6):705-714.

5. Bartha JL, Illanes S, González-Bugatto F, et al. Maternal serum transformed a-fetoprotein levels in women with intrauterine growth retardation. Fetal Diagn Ther. 2007;22(4):294-298.

6. Heinonen S, Ryynänen M, Kirkinen P. The effects on fetal development of high a-fetoprotein and maternal smoking. Am J Public Health. 1999;89(4):561-563

7. Krause TG, Christens P, Wohlfahrt J, et al. Second-trimester maternal serum alpha-fetoprotein and risk of adverse pregnancy outcome. Obstet Gynecol. 200;97(2):277-282.

8. Smith GCS, Shah I, Crossley JA, et al. Pregnancy-associated plasma protein A and alpha-fetoprotein and prediction of adverse perinatal outcome. Obstet Gynecol. 2006;107:161-166.

9. Cho S, Durfee KK, Keel BA, et al. Perinatal outcomes in a prospective matched pair study of pregnancy and unexplained elevated or low AFP screening. Journal of Perinatal Medicine-Official Journal of the WAPM.1997;25(6):476-483.

10. Jelliffe-Pawlowski LL, Baer RJ, Currier RJ. Second trimester serum predictors of preterm birth in a population-based sample of low-risk pregnancies. Prenat Diagn. 2010;30(8):727-733.

11. Purdie DW, Young JL, Guthrie KA, et al. Fetal growth achievement and elevated maternal serum a-fetoprotein. BJOG Int $J$ Obstet Gynaecol.1983;90(5):433-436.

12. Tikkanen M, Hämäläinen E, Nuutila M, et al. Elevated maternal secondtrimester serum alpha-fetoprotein as a risk factor for placental abruption. Prenat Diagn. 2007;27(3):240-243.

13. Flenady V, Koopmans L, Middleton P, et al. Major risk factors for stillbirth in high-income countries: a systematic review and meta-analysis. Lancet. 2011;377(9774):1331-1340.

14. Talip Q, Theron G, Steyn W, et al. Total perinatally related losses at Tygerberg hospital -A comparison between 1986, 1993 and 2006. S Afr Med J. 2010;100:250-253.

15. Odendaal HJ, Dukes KA, Elliott AJ. Stillbirth and Prenatal Exposure to Alcohol and Cigarettes: Report of the Safe Passage Study.

16. Aliyu MH, Lynch O, Nana PN, et al. Alcohol consumption during pregnancy and risk of placental abruption and placenta previa. Matern Child Health J. 2011;15(5):670-676.

17. Du Toit MM, Smith M, Odendaal HJ. The role of prenatal alcohol exposure in abruptio placentae. S Afr Med J. 2010;100(12): 832-835.

18. Marbury MC, Linn S, Monson R, et al. The association of alcohol consumption with outcome of pregnancy. Am $J$ Public Health 1983;73(10):1165-1168.
19. Patra J, Bakker R, Irving H, et al. Dose-response relationship between alcohol consumption before and during pregnancy and the risks of low birthweight, preterm birth and small for gestational age (SGA) - a systematic review and meta-analyses. BJOG: Int J Obstet Gynaecol. 2011;118(12):1411-1421.

20. Aliyu MH, Salihu HM, Wilson RE, Kirby RS. Prenatal smoking and risk of intrapartum stillbirth. Arch Environ Occup Health. 2007;62(2):87-92.

21. Aliyu MH, Salihu HM, Alio AP, et al. Prenatal smoking among adolescents and risk of fetal demise before and during labor. $J$ Pediatr Adolesc Gynecol. 2010;23(3):129-135.

22. Ashmead G. Smoking and pregnancy. The Journal of Maternal-Fetal \& Neonatal Medicine. 2003;14(5):297-304.

23. Dukes KA, Burd L, Elliott AJ, et al. The safe passage study: Design, methods, recruitment, and follow-up approach. Paediatr Perinat Epidemiol. 2014;28(5):455-465.

24. Jauniaux E, Suri S, Muttukrishna S. Evaluation of the impact of maternal smoking on ultrasound and endocrinological markers of first trimester placentation. Early Hum Dev. 2013;89(9):777-780.

25. Odendaal HJ, Elliott A, Kinney HC, et al. Consent for autopsy research for unexpected death in early life. Obstet Gynecol 2011;117(1):167-171.

26. Boyd TK, Wright CA, Odendaal HJ, et al. The stillbirth classification system for the Safe Passage Study: Incorporating mechanism, etiology, and recurrence. Pediatr Dev Pathol. 2017;20(2):120-132.

27. Dukes K, Tripp T, Petersen J, et al. A modified Timeline Followback assessment to capture alcohol exposure in pregnant women: Application in the Safe Passage Study. Alcohol. 2017;62:17-27.

28. Dukes K, Tripp T, Willinger M, et al. Drinking and smoking patterns during pregnancy: Development of group-based trajectories in the Safe Passage Study. Alcohol. 2017;62:49-60.

29. Villar J, Papageorghiou AT, Pang R, et al. The likeness of fetal growth and newborn size across non-isolated populations in the INTERGROWTH21st Project: the Fetal Growth Longitudinal Study and Newborn CrossSectional Study. Lancet Diabetes Endocrinol. 2014;2(10):781-792.

30. Halmesmaki E, Autti I, Granstrom M, et al. Prediction of fetal alcohol syndrome by maternal alpha fetoprotein, human placental lactogen and pregnancy specific beta 1-glycoprotein. Alcohol. 1987;1:473-476.

31. Spencer K. The influence of smoking on maternal serum AFP and free beta hCG levels and the impact on screening for Down syndrome. Prenat Diagn. 1998;18(3):225-234.

32. Urato AC, Quinn R, Pulkkinen A, et al. Maternal smoking during pregnancy and false positive maternal serum alpha-fetoprotein (MSAFP) screening for open neural tube defects. Prenat Diagn. 2008;28(8):778-780.

33. Zhang J, Lambert-Messerlian G, et al. Impact of smoking on maternal serum markers and prenatal screening in the first and second trimesters. Prenat Diagn. 2011;31(6):583-588

34. Filis P, Nagrath N, Fraser M, et al. Maternal smoking dysregulates protein expression in second trimester human fetal livers in a sex-specific manner. J Clin Endocrinol Metab. 2015;100(6):E861-870.

35. Spencer K. Second-trimester prenatal screening for Down syndrome and the relationship of maternal serum biochemical markers to pregnancy complications with adverse outcome. Prenat Diagn. 2000;20(8):652-656.

36. Bazzett LB, Yaron Y, O'Brien JE, et al. Fetal gender impact on multiplemarker screening results. Am J Med Genet. 1998;76(5):369-371. 
37. Sultana T, Karim MN, Ahmed T, et al. Assessment of under nutrition of Bangladeshi adults using anthropometry: can body mass index be replaced by mid-upper-arm-circumference? PloS one. 2015;10(4):e0121456.

38. Dehghani-Firouzabadi R, Tayebi N, Ghasemi N, et al. The association between second-trimester maternal serum alpha-fetoprotein in 14-22 weeks and adverse pregnancy outcome. Acta Med Iran. 2010;48(4):234-238

39. Van Rijn M, Van Der Schouw YT, Hagenaars AM, et al. Adverse obstetric outcome in low- and high-risk pregnancies: Predictive value of maternal serum screening. Obstet Gynecol. 1999;94(6):929-934.

40. Cho S, Durfee KK, Keel BA, et al. Perinatal outcomes in a prospective matched pair study of pregnancy and unexplained elevated or low AFP screening. J Perinat Med. 1997;25(6):476-483.
41. Geldenhuys E, Coldrey J, Wright C, et al. Fetal foot length at delivery as a tool for determining gestation length in non-macerated stillbirths. Int $J$ Gynaecol Obstetrics. 2017;138(1):107-112.

42. Lesmes C, Gallo DM, Gonzalez R, etal. Prediction of small-for-gestationalage neonates: Screening by maternal serum biochemical markers at 19-24 weeks. Ultrasound Obstet Gynecol. 2015;46(3):341-349.

43. Muller F, Dreux S, Oury J, et al. Down syndrome maternal serum marker screening after 18 weeks' gestation: a country wide study. Am J Obstet Gynecol. 2013;208(5):397. 